\title{
Mechanical and Electrophysiological Properties of the Sarcolemma of Muscle Fibers in Two Murine Models of Muscle Dystrophy: Col6a 1-/- and Mdx
}

\author{
M. Canato, ${ }^{1}$ M. Dal Maschio, ${ }^{1,2}$ F. Sbrana, ${ }^{3,4}$ R. Raiteri, ${ }^{4}$ C. Reggiani, ${ }^{1}$ S. Vassanelli, ${ }^{1}$ \\ and A. Megighian ${ }^{1}$ \\ ${ }^{1}$ Department of Human Anatomy and Physiology, University of Padova, 35131 Padova, Italy \\ ${ }^{2}$ Department of Neuroscience and Brain Technology, IIT, 16163 Genova, Italy \\ ${ }^{3}$ CSDC-Department of Physics, University of Firenze, 50019 Sesto Fiorentino, Italy \\ ${ }^{4}$ Department of Biophysical and Electronic Engineering, University of Genova, 16145 Genova, Italy
}

Correspondence should be addressed to C. Reggiani, carlo.reggiani@unipd.it

Received 31 October 2009; Accepted 31 January 2010

Academic Editor: Henk L. M. Granzier

Copyright (C) 2010 M. Canato et al. This is an open access article distributed under the Creative Commons Attribution License, which permits unrestricted use, distribution, and reproduction in any medium, provided the original work is properly cited.

\begin{abstract}
This study aimed to analyse the sarcolemma of Col6a1-/- fibers in comparison with wild type and mdx fibers, taken as positive control in view of the known structural and functional alterations of their membranes. Structural and mechanical properties were studied in single muscle fibers prepared from FDB muscle using atomic force microscopy (AFM) and conventional electrophysiological techniques to measure ionic conductance and capacitance. While the sarcolemma topography was preserved in both types of dystrophic fibers, membrane elasticity was significantly reduced in Col6a1-/- and increased in mdx fibers. In the membrane of Col6a1-/- fibers ionic conductance was increased likely due to an increased leakage, whereas capacitance was reduced, and the action potential (ap) depolarization rate was reduced. The picture emerging from experiments on fibers in culture was consistent with that obtained on intact freshly dissected muscle. Mdx fibers in culture showed a reduction of both membrane conductance and capacitance. In contrast, in mdx intact FDB muscle resting conductance was increased while resting potential and ap depolarization rate were reduced, likely indicating the presence of a consistent population of severely altered fibers which disappear during the culture preparation.
\end{abstract}

\section{Introduction}

Bethlem myopathy and Ulrich congenital dystrophy are inherited diseases which share a common origin in mutations of collagen VI (COL VI) encoding gene leading to a defective extracellular matrix composition $[1,2]$. The clinical symptoms are very different as Bethlem myopathy is a relatively mild and slowly progressive disorder characterized by early onset, weakness, and wasting of skeletal muscles, and multiple joint contractures, whereas Ulrich congenital muscle dystrophy (UCMD) is a severe disorder characterized by generalized and progressive muscle wasting and weakness, and by the coexistence of proximal joint contractures and striking distal hyper-extensibility. The link between altered extracellular matrix composition and muscle fiber degeneration is not yet completely understood and the study of Col6a1-/- mice, is expected to give an important contribution. Col6al-/- mice [3] carry a null mutation of the $\alpha 1$ chain of COL VI and exhibit a phenotype which is reminiscent of some features present in human Bethlem myopathy and UCMD. In particular, muscles of Col6a1-/- mice share with muscles of humans affected by COL VI mutations the high rate of apoptosis with apoptotic nuclei being 5-6 fold more abundant than in muscles of wild type (wt) mice [3]. A mitochondrial mechanism based on increased opening probability of the permeability transition pore (PTP) within the mitochondrial inner membrane has been proposed and confirmed by the finding that treatment of Col6a1-/- mice with Cyclosporin A, a PTP inhibitor, rescued the mutant dystrophic muscle 
fibers [4]. More recently similar results have been obtained also in UCMD patients [5]. In spite of such important advancements, the signalling pathway connecting lack of COL VI and mitochondrial dysfunction remains unknown. Ultrastructural alterations of sarcoplasmic reticulum and mitochondria and abnormal variations in cytosolic calcium in the presence of the mitochondrial inhibitor olygomycin might suggest a possible involvement of calcium [4]. Integrin-mediated effects on mitochondrial function [6] have been considered. A possible role of NG2 which is significantly decreased in patients with COL VI mutation as well as in Col6a1-/- mice, has been also proposed [7].

In view of the uncertainty on the pathogenic mechanisms of the myopathy induced by the lack of COLVI, we sought to investigate the structural and functional properties of the sarcolemma. Actually, the sarcolemma represents the barrier and, at the same time, the link between extracellular matrix and intracellular environment. It is, therefore, possible that the first effects of the lack of a structural component of the ECM, such as COL VI, can be detected at the sarcolemma. ECM molecules are linked to the cell cytoskeleton through large multimeric complexes where proteins like integrins [8] and dystroglycans [9] play a pivotal role. These protein complexes provide the connection with the intracellular cytoskeleton but are also involved in controlling intracellular signalling pathways and in the recruitment and clustering ion channels, thereby regulating the electrophysiological response of the cell membrane [10].

The present study was designed to assess whether biophysical properties of the sarcolemma are altered in muscle fibers of mice lacking COL VI. To this end we combined a structural and mechanical exploration based on Atomic Force Microscopy (AFM) with the analysis of the basic electrophysiological features of skeletal muscle fibers both in freshly dissected muscles and in culture. Wild type and $\mathrm{mdx}$ muscle fibers were utilized as controls. Actually, mdx muscle fibers can be considered a positive control as alterations in sarcolemmal electrophysiological and mechanical properties have been reported in previous studies $[11,12]$.

\section{Materials and Methods}

2.1. Animals. Experiments were carried out on young adult (1-2 month old) Col6a1-/- mice, mdx mice and the related wild type mice $\mathrm{C} 57 \mathrm{BL} / 6$. Wild type and $\mathrm{mdx}$ mice were purchased at Charles River Italia (Calco, Italy). Col6a1-/- mice (kind gift from Professor Paolo Bonaldo, University of Padova) were obtained as previously described using a targeting vector containing a neomycin resistance cassette in the second exon to inactivate the colbal gene in embryonic stem cells [3]. The use of the animals and the experimental protocol was approved by the Department Ethical Committee and the University Ethical Commission (CEASA, Comitato Etico di Ateneo per la Sperimentazione sugli Animali). All efforts were made to minimize animal suffering and to keep low the number of animals necessary to obtain reliable data.
Mice were killed by cervical dislocation and FDB (Flexor Digitorum Brevis) muscles were quickly dissected out and pinned on the sylgard (Sylgard 184, Down Corning, Milano, Italy) coated bottom of a Petri dish. Muscles were continuously perfused with Tyrode solution bubbled with a 95\% $\mathrm{O}_{2}$ $5 \% \mathrm{CO}_{2}$ gas mixture.

2.2. Preparation and Culture of Muscle Fibers. Single muscle fibers were enzymatically isolated from FDB following a protocol derived from that described by Head [13]. After dissection FDB muscles were placed in a Petri dish filled with tyrode solution (mM: $\mathrm{NaCl} 140, \mathrm{KCl} 2, \mathrm{CaCl}_{2}$ 2, Hepes 10, and glucose 5) containing 0,2\% tipe I collagenase (C0130, Sigma-Aldrich, Milano, Italy) and $10 \%$ foetal bovine serum (FBS) for 1 hour at $4^{\circ} \mathrm{C}$ and then for another hour at $37^{\circ} \mathrm{C}$ in a water-saturated incubator with $5 \% \mathrm{CO}_{2}$. After three washes in Tyrode containing 10\% FBS to block the collagenase effect and stabilize the fibers, the muscles were gently and repeatedly sucked in and out a fire-polished Pasteur pipettes of different diameters $(30-300 \mu \mathrm{m})$ inside a glass Falcon until muscle fibers were dissociated. Dissociated fibers were plated on coverslips covered with mouse laminin which induced fiber attachment within 1 hour. Coverslips were kept in small Petri dishes filled with Tyrode supplemented with $10 \%$ FBS and $1 \%$ penicillin-streptomycinanphotericin. Culture dishes were kept in incubator at $37^{\circ} \mathrm{C}$, $5 \% \mathrm{CO}_{2}$.

2.3. Atomic Force Miscroscopy (AFM). An integrated AFM/inverted optical microscope system (5500ILM AFM, Agilent Technologies equipped with an Olympus IX70 inverted microscope) was used for imaging and elasticity measurements. This allowed to perform simultaneous optical (phase contrast) and AFM imaging on living or fixed (paraphormaldeyde 4\% for $20 \mathrm{~min}$ ) fibers. Fibers were examined 6 days after dissociation. The AFM was equipped with a $100-\mu \mathrm{m}$ XY scanner. Triangular silicon nitride cantilevers with a spring constant $\mathrm{K}=0.012 \mathrm{~N} / \mathrm{m}$ and sharp pyramidal tips (Veeco, Santa Barbara, CA) were employed. The spring constant of each cantilever was determined by the thermal noise method which ensures a level of accuracy of $10 \%-15 \%$ [14].

Topography and elasticity images were acquired by recording force versus distance curves on a regular grid of $64 \times 64$ points over an $5 \times 5 \mu \mathrm{m}^{2}$ area. Each force curve was taken at a rate of $2 \mathrm{~Hz}$, applying a max load of $1 \mathrm{nN}$ corresponding to a max indentation of $100 \mathrm{~nm}$. The time to obtain a full force map was therefore approximately 50 minutes. For each point of the grid (i.e., for each curve) the values of the sample height at $500 \mathrm{pN}$ load and of the elasticity were calculated. To exclude any possible contribution from plastic (i.e., permanent) deformation, only data from the unloading (retracting) part of each curve were considered and converted into a load versus indentation curve. The elasticity value (i.e. Young's modulus) E, was calculated at the indentation of $100 \mathrm{~nm}$ using the Oliver's and Pharr model for conical tips: [15]: $E=\pi^{0.5} / 2\left(1-\nu^{2}\right) S / A^{0.5}$, where $v$ is the Poisson's ratio, $\mathrm{S}$ the slope or contact stiffness, 
and $\mathrm{A}$ is the effective projected area of the indenter tip on the sample. We assumed a value for the Poisson's ratio $v=$ 0.5 and approximated the tip geometry corresponding to a square-based pyramid with a $35^{\circ}$ aperture angle, with a conical shape with the same angle of aperture.

The calculation of the average value $E$ over the map of $64 \times 64=4096$ curves was performed using a custom analysis software developed in LabView (National Instruments, Austin, Texas): for each force curve in a map, the slope $S$ of the unloading curve at the maximum load is automatically calculated. The histogram of the calculated $S$ values for each single map showed one peak, and it was fitted with a Gaussian curve. $30 \%$ of the curves around the fit mean (typically 1000 curves) are then averaged. The averaged curve was used to calculate the $E$ value.

2.4. Electrophysiology. Electrophysiological properties were investigated using intracellular electrodes on single enzymatically-dissociated fibers after 6 days of culture and on intact muscles dissected and pinned on the bottom of a sylgardcoated Petri dish. In both cases, experiments were carried out at room temperature $\left(20^{\circ}-22^{\circ} \mathrm{C}\right)$. Whole FDB muscles or single dissociated fibers were bathed in normal physiological (Tyrode) saline with the following composition in $\mathrm{mM}: \mathrm{NaCl}$ $140, \mathrm{KCl} 2, \mathrm{CaCl}_{2} 2$, HEPES 10, glucose 5, and $\mathrm{pH}$ 7.4. In case of whole muscles, solution was continuously bubbled with $95 \% \mathrm{O}_{2}$.

Fiber action potential was intracellularly recorded using a bridge amplifier (SEC 10L, NPI, Germany) and currentclamp approach. Amplified data were then digitized at $25 \mathrm{kHz}$ with an ADC board (National Instruments PCI6071E or Axon Digidata 1200) and stored in a PC for offline analysis using appropriate software (WinWCP, Strathclyde University; pClamp6, Axon Instruments). After the insertion of the micropipette $(0.5 \mu \mathrm{m}$ tip diameter; filled with $3 \mathrm{M} \mathrm{KCl}$; $10 \mathrm{Mohm}$ resistance), membrane potential was clamped to $-100 \mathrm{mV}$ by injecting a negative current.

In order to determine fiber excitability, once membrane potential was set at $-100 \mathrm{mV}$, fibers were stimulated using positive square-wave current pulses $(500 \mathrm{~ms}$ duration) of increasing intensity until an action potential was elicited. For the first action potential the amplitude in $\mathrm{mV}$, the threshold current, in $\mathrm{nA}$, and the latency of action potential, that is, the delay from the beginning of the current pulse to the onset of an action potential at threshold, in ms, were measured (see [14]). The amplitude and the rates of depolarization and repolarization were then measured in subsequent action potentials.

In order to investigate passive membrane properties, the cell membrane charging process in response to a negative current pulse (200ms duration; $1 \mathrm{nA}$ amplitude) from a holding potential of $-100 \mathrm{mV}$, was analysed. A direct measurement of membrane conductance was made from the change in potential at the end of the hyperpolarizing pulse when membrane potential was stable. In a set of experiments anthracene 9-carboxylate (9-AC) (Sigma-Aldrich, Milano, Italy) was added to to bath $(100 \mu \mathrm{M})$ to block chloride currents.
Images of the fibers studied in electrophysiological experiments were taken at 20 and $300 \times$ magnification and used to measure diameters and length and to calculate surface area, assuming a cylindrical shape.

To identify membrane capacitance and conductance, the membrane potential transients were described in terms of a time-independent component $\left(R_{i}\right)$ which follows the current step within $5 \mathrm{~ms}$ and a time dependent component which was analysed in terms of a two parameters model describing the slow charging process of the cell membrane with the equation

$$
\begin{aligned}
\frac{V_{M}}{I_{\mathrm{COM}}} & =R_{M} \cdot\left(1-\exp \left(-\frac{t}{C_{M}} \cdot R_{M}\right)\right) \\
& =R_{M} \cdot\left(1-\exp \left(-\frac{t}{\tau_{M}}\right)\right),
\end{aligned}
$$

derived from a simple membrane analogue with membrane capacitance $C_{M}$ in parallel with membrane resistance $R_{M}$. From the parameters $R_{M}$ and $\tau_{M}$ obtained with minimization of the squared mean error, the value for the membrane capacity $C_{M}$ was calculated and normalized to the apparent cell surface. The time independent component was identified as the leakage branch of the model.

2.5. Statistical Analysis and Curve Fitting. Data were expressed as mean and standard error. Comparisons between Col6a1-/-, mdx, and wild type muscle fibers were done using one way variance analysis followed by Newman-Keuls test. Differences were considered significant at $P<.05$.

The fitting of the membrane potential charging process was performed by a Labview (National Instruments, Austin, Texas) module of Levenberg-Marquardt algorithm of minimization applied to the root mean error calculated from the experimental dataset.

\section{Results}

3.1. Viability of Col6a1-/- Fibers in Culture. Single FDB fibers, dissociated and cultured as described in the Materials and Methods, were monitored up to 12 days. From the enzymatic dissociation of each FDB muscle approximately 300-400 fibers were obtained. As can be seen in Figure 1, both Col6a1-/- mutant and wild-type fibers showed a progressive loss of viability with the time, while the cultures of dystrophin lacking mdx fibers showed an initial drop during the first day. This suggests that all Col6a1-/fibers damaged or apoptotic were removed during the preparation and only the fibers in good conditions could survive the collagenase treatment. Apparently, such fibers from Col6a 1-/- mice did not show any significant alteration of viability or shorter survival time in culture conditions, at least within the 12 days time window considered. By contrast, for $\mathrm{mdx}$ fibers a severe selection occurred in the first day after dissociation and only a fraction of fibers were sufficiently viable to survive for many days in culture. Experiments were performed at day 6 , when cells had recovered from dissociation and were capable to generate action potentials upon stimulation. At day 6, the measurements on enlarged 
video images of randomly chosen groups of cells showed only minor and not significant differences in length and in diameter (Figure 1). Staining with Hoechst nuclear dye did not provide evidence of centrally located nuclei, thus suggesting that no regenerating fibers were present in the cultures (not shown).

3.2. Sarcolemma Topography and Elasticity Studied with Atomic Force Miscroscopy (AFM). Sarcolemma topography images of wt, Col6a1-/- and mdx fibers were obtained with AFM on living fibers and after fixation with paraphormaldeyde. In paraphormaldeyde-fixed fibers (Figure 2) a periodic pattern of foldings perpendicular to the fiber long axis was clearly detectable and showed a periodicity of approximately $2 \mu \mathrm{m}(1.98 \pm 0.08 \mu \mathrm{m}$ in wt and $1.88 \pm 0.15 \mu \mathrm{m}$ in Col6a1 $-/-$ and $1.78 \pm 0.12 \mu \mathrm{m} \mathrm{mdx} \mathrm{fibers}$ resp., not significantly different). As demonstrated in our previous study [15], the periodicity of the major sarcolemmal folds corresponds to the periodicity of the sarcomeres, as determined by phase contrast microscopy, and the major folds correspond to the Z-lines. Interestingly, in all three fiber groups a less evident but consistent folding was detectable between the major folds. Such intermediate periodic feature might correspond to the M-line [15]. The depth of the major foldings range between 50 and $150 \mathrm{~nm}$, without consistent differences between the three groups of fibers. Thus, no clear difference in the general sarcolemmal architecture was detectable with AFM topography.

Nine Col6a1-/- fibers, $14 \mathrm{mdx}$ fibers, and $18 \mathrm{wt}$ fibers were considered for elasticity analysis with AFM (see Figure 3). The fibers were cultured in Petri dishes and examined without fixation, as described in the Methods. For each fiber, two or three topography and elasticity maps were recorded for a total of 18 maps for Col6a1-/-, 21 maps for $\mathrm{mdx}$, and 29 maps for wild type. In the same fibers, sarcomere periodicity was optically measured with phase contrast and average $( \pm$ s.e.) sarcomere length was found to be $1.83 \pm 0.02 \mu \mathrm{m}$ in Col6a1-/-, $1.78 \pm 0.01$ in $\mathrm{mdx}$ and $1.90 \pm 0.01$ in wild type fibers, values in good agreement with the results obtained for folding periodicity in fixed fibers. The differences, although very small, were statistically significant $(P<.05)$. An example of phase contrast image of a Col6a-/- fiber cultured for 6 days in contact with the AFM cantilever is shown in Figure 3(g).

Topography and elasticity maps were obtained as described in the Methods. Examples of such maps for three fibers: wild type, Col6a1-/- and $\mathrm{mdx}$, respectively, are shown in Figures 3(a)-3(f). Periodic features are detectable both in the topography maps (Figures 3(a), 3(c) 3(e)), and elasticity maps (Figures 3(b), 3(d), 3(f)), in agreement with our previous observations [15]. The topography and the elasticity map in each row ( $a$ and $b, c$ and $d, e$ and $f$ ) refer to the same region of a given fiber (wild type a, b; Col6a1-/c, d; mdx e, f). Interestingly, as can be appreciated by the grey scale where white corresponds either to stiffer (higher elasticity or Young Modulus value) regions or to more elevated regions, the tops of the foldings roughly correspond to the stiffer parts of the sarcolemma. The average elasticity values are shown in the histogram in Figure $3(\mathrm{~g})$ and, as can be seen, the surface of Col6a1-/- fibers was significantly less stiff than the surface of wild type fibers, while the average stiffness of the surface of $\mathrm{mdx}$ fibers was significantly greater than that of wild type fibers.

3.3. Resting Potential and Action Potential Measured in $F D B$ Fibers in Culture. Intracellular electrode recordings showed that resting membrane potential was similar in the mutant and wild type fibers (Figure 4(a)). Depolarizing current steps of increasing amplitude were applied until an action potential was elicited. Although some fibers from mutant mice were unexcitable (less than 20\%), no significant difference of voltage threshold for action potentials between wild type and dystrophic fibers (with preserved excitability) was observed. The action potential rising rate and amplitude were also measured. Whereas the amplitude was identical in wild type, $\mathrm{mdx}$, and Col6a1-/- fibers, the depolarization rate was significantly lower in Col6a1-/- fibers (Figures 4(b) and $4(\mathrm{c}))$.

\subsection{Membrane Conductance and Capacitance of FDB Fibers} in Resting Potential Conditions. The membrane conductance at resting potential was measured by applying to the fibers a hyperpolarizing current step, as described in the Methods. Average conductance normalized to fiber surface was higher in Col6a1-/- than in wild type fibers, while in $\mathrm{mdx}$ fibers was lower than in wild type fibers, as shown in Figure 4(d).

Since in mammalian skeletal muscle fibers the main determinant of resting sarcolemma conductance is chloride conductance [16], we repeated the measurement after blocking chloride channels with 9-anthracene carboxylic acid (9AC) [17] to check whether the difference between wild type and Col6a1-/- could be removed. As shown in Figure 4(e), 9-AC caused a significant reduction of membrane conductance both in wild type and Col6al-/- fibers. The reduction was, however, greater in wild type fibers $(-40 \%)$ than in Col6a1-/- fibers $(-20 \%)$, thus suggesting that the higher conductance found in Col6a1-/- was likely not due to chloride channels.

To further investigate the source of the different values of membrane conductance of Col6a1-/- and $\mathrm{mdx}$ fibers, shown in Figure 4(d), the kinetics of intracellular voltage changes in response to a hyperpolarizing step was interpolated by (1) corresponding to the model described in the Methods (Figure 5). The parameters calculated by the best fitting provided an estimate of: (i) the resting conductance $\left(R_{M}\right)$, (ii) the capacitance $\left(C_{M}\right)$, and (iii) the instantaneous or time independent conductance $\left(R_{i}\right)$, identified as the membrane leakage. The average values of the three parameters, obtained in wild-type and mutant fibers after 6 days in culture are reported in Figure 5, together with the values of the time constant $\tau_{M}$ of the membrane charging process. As can be seen in Figure 5(c), the time constants were significantly different and the lower value observed in Col6a1-/ - fibers was attributed by the model to a lower capacitance of the membrane associated with similar conductance values (Figures 5(e) and 5(f)). By contrast, mdx 


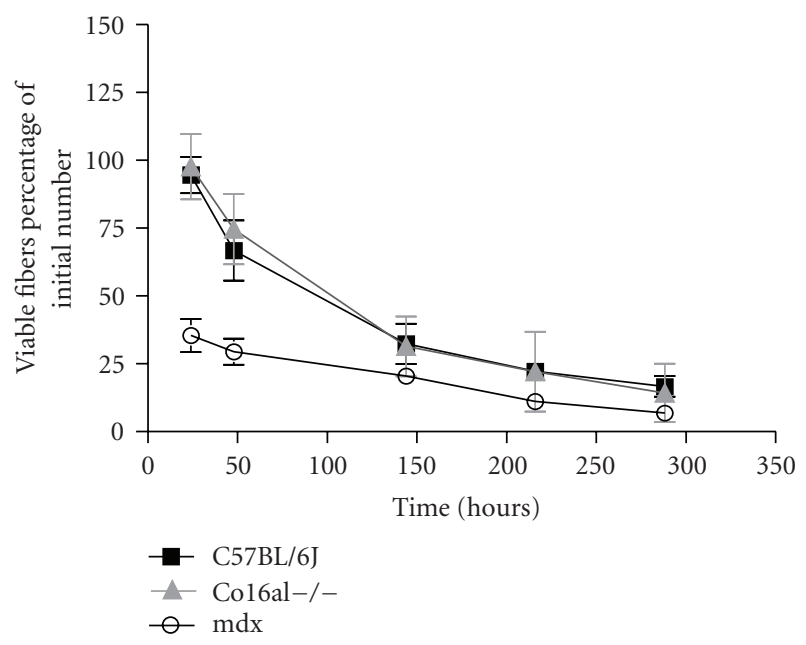

(a)

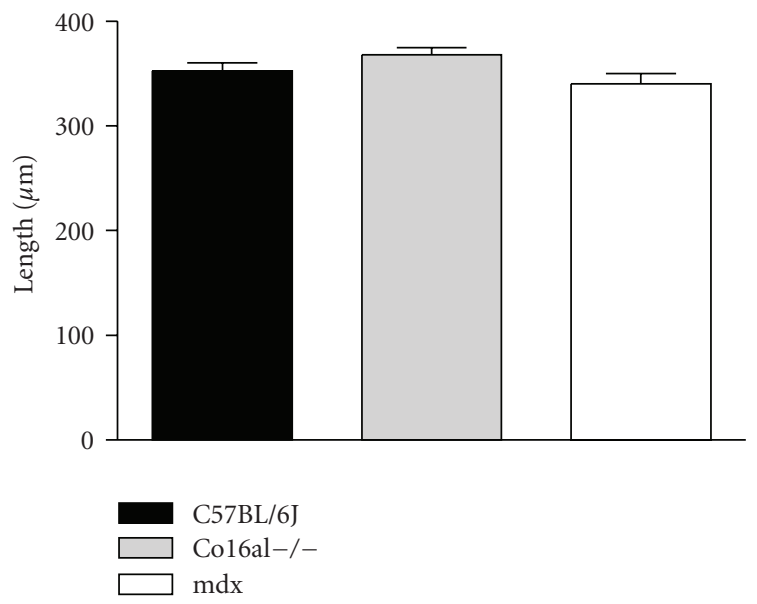

(b)

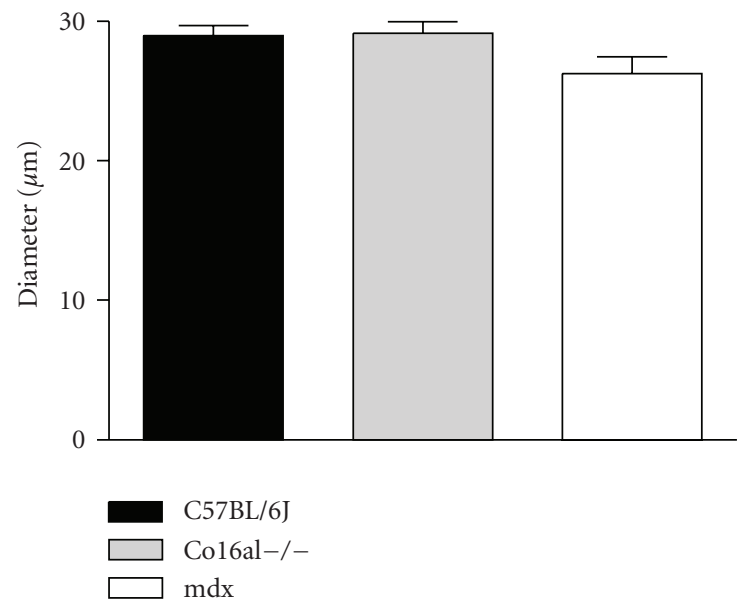

(c)

Figure 1: Survival curve of muscle fibers in culture. Time 0 corresponds to muscle dissection and the fraction of the surviving fibers is expressed in \% of the fibers attached on the bottom of the Petri dishes 24 hours after dissection. No difference is detectable in the survival between wild type and Col6a1-/- fibers, while the survival of mdx fibers is significantly lower. 

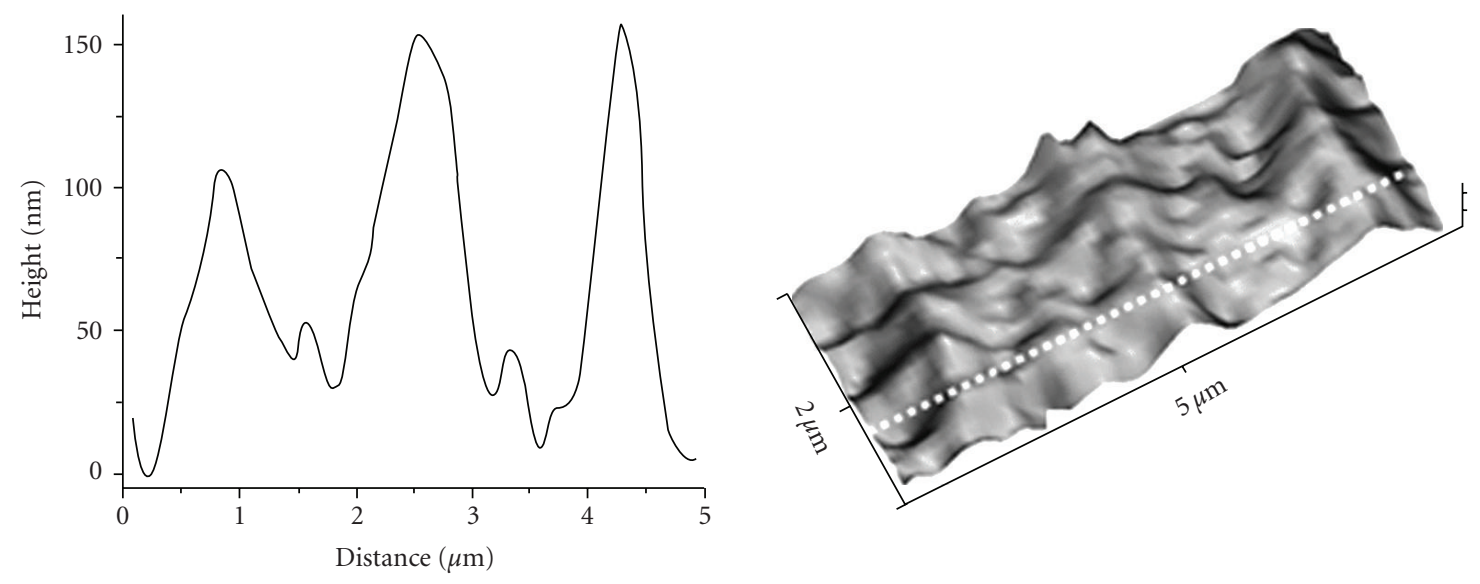

(a)
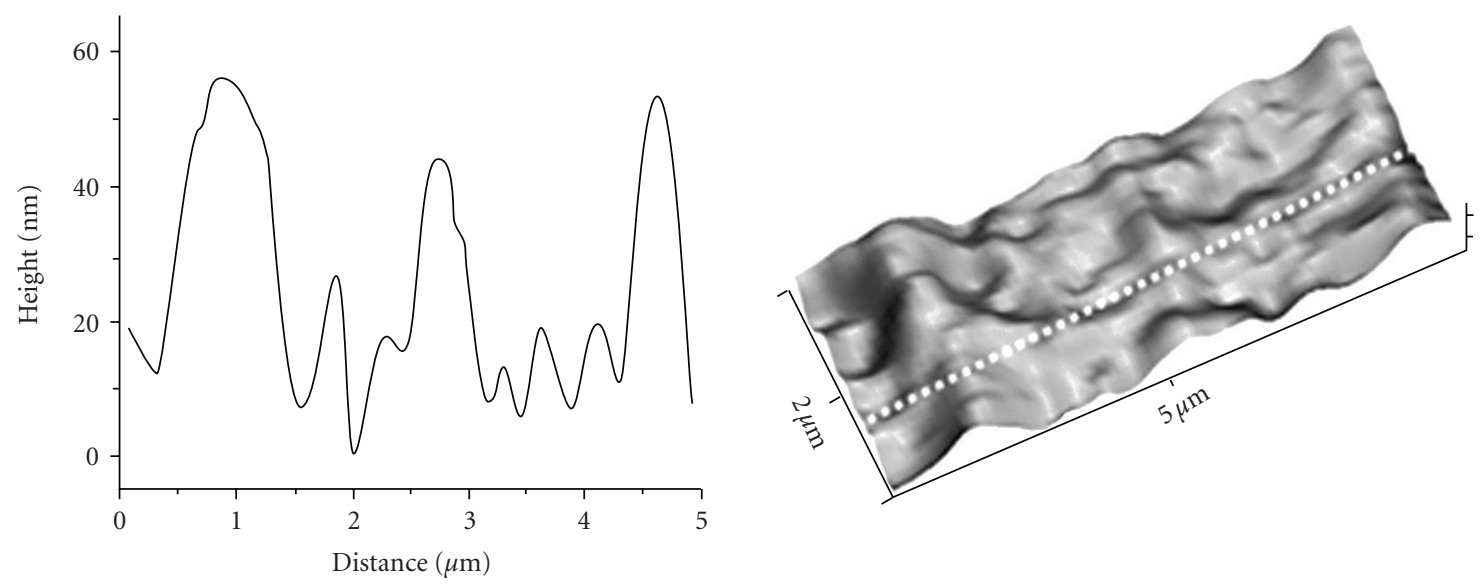

(b)
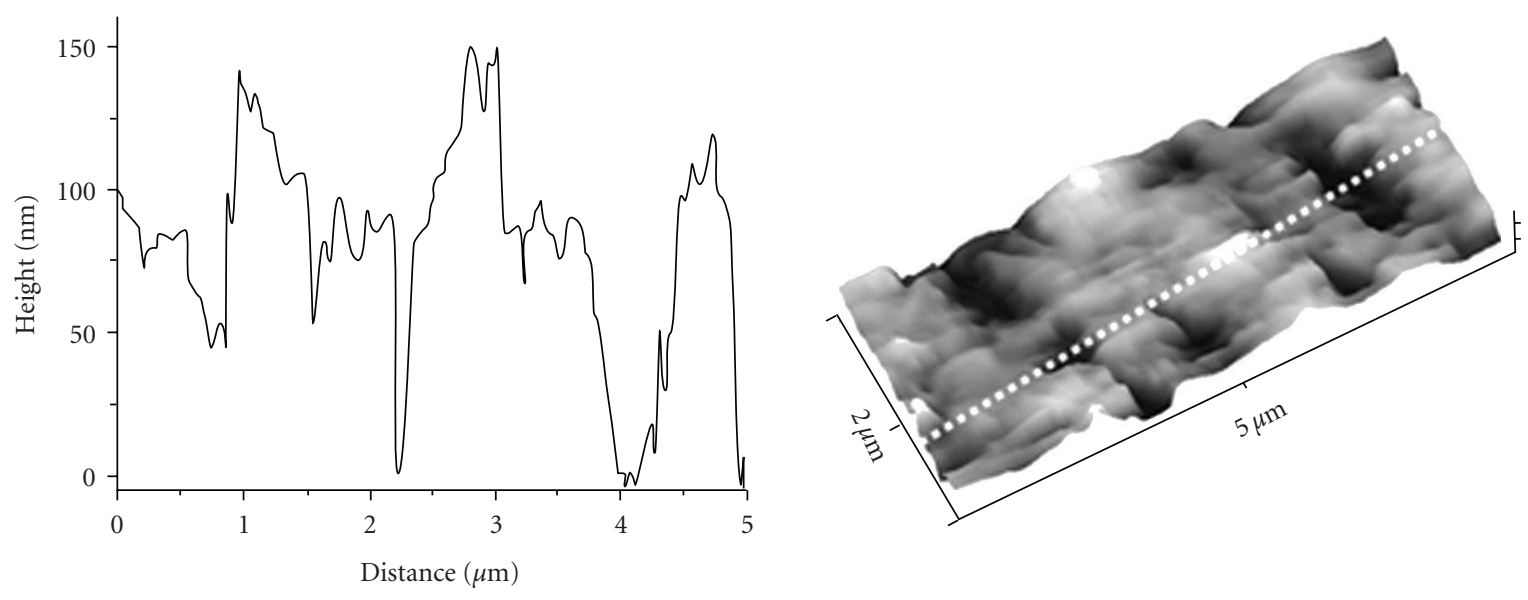

(c)

FIGURE 2: AFM topography (contact mode) of fixed fibers: wild type (a), Col6al-/- (b) and mdx (c) fibers. Two sets of foldings with a periodic pattern can be easily recognized in all three fiber types. The periodicity of the greater foldings, likely in phase with Z-line is about $2 \mu \mathrm{m}$, and the distance between the greater and the smaller foldings is about $1 \mu \mathrm{m}$. The profiles reported in the diagrams on the left are obtained along the dashed lines in the $3 \mathrm{D}$ images on the right side. 


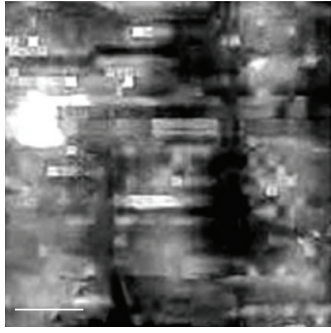

(a)

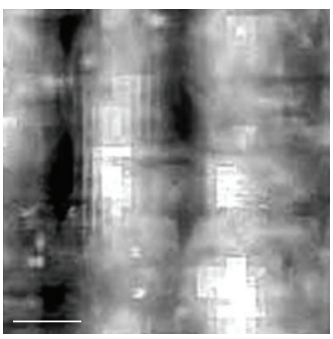

(c)

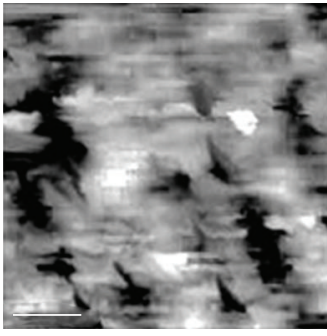

(e)
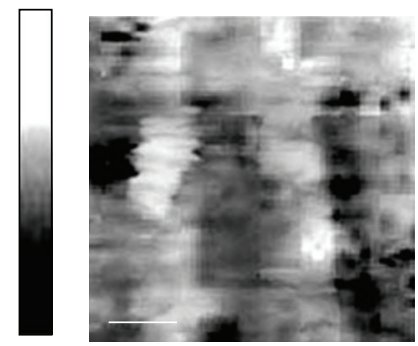

(b)
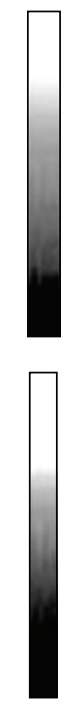

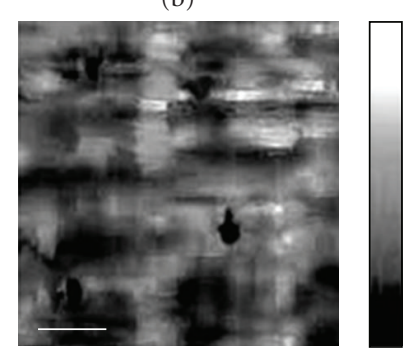

(d)

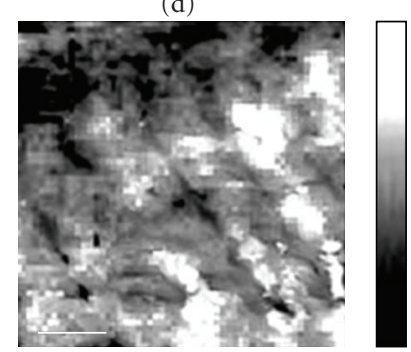

(f)
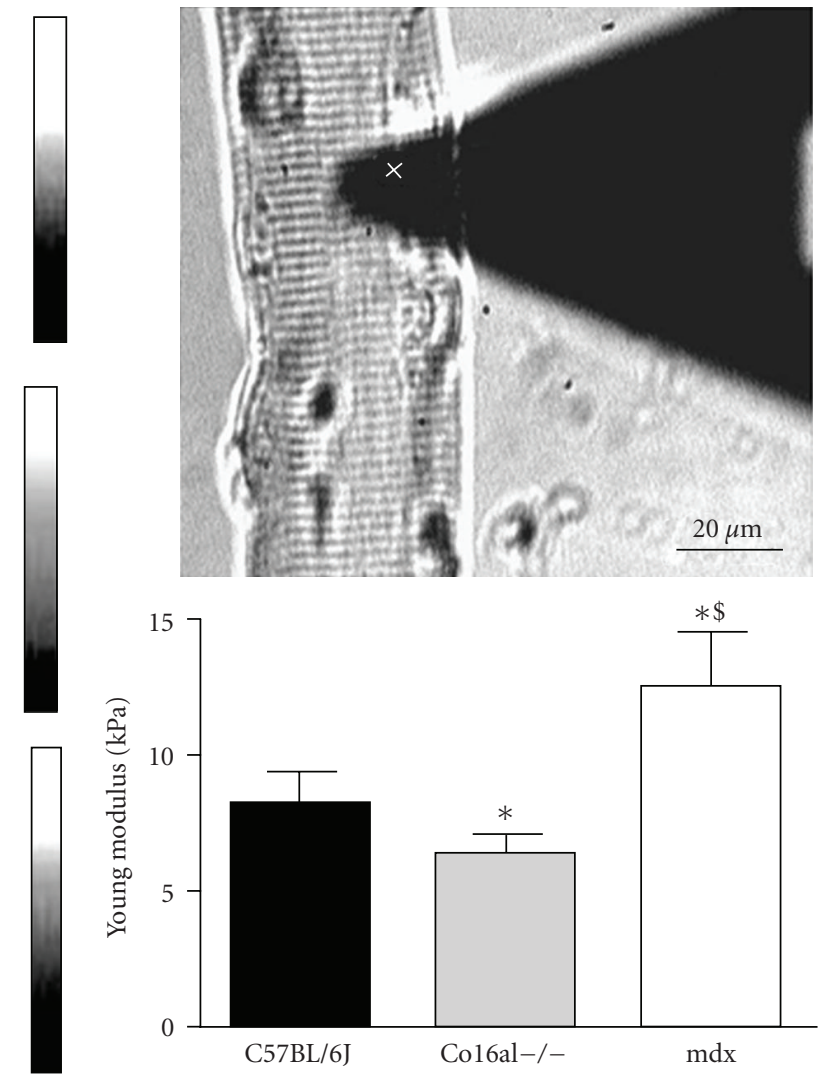

(g)

Figure 3: Topography (a, c, and e) and elasticity (b, d, and f) images obtained from a force map $(64 \times 64$ force curves, max applied force $=$ $1 \mathrm{nN})$ taken on a wild type $(\mathrm{a}, \mathrm{b})$, Col6a $1-/-(\mathrm{c}, \mathrm{d})$ and $\mathrm{mdx}(\mathrm{e}, \mathrm{f})$ fibers. The rectangles in the middle indicate in a grey scale (increasing from black to white) the depth with a range of $210 \mathrm{~nm}$ in the topography images and the elasticity with a range of $1 \mathrm{kPa}$ in the elasticity images. The white segments in the left lower corner corresponds to $1 \mu \mathrm{m}$. g: Phase contrast image of a Colba1-/- fiber in culture with the triangular AFM cantilever on top of it. The white cross indicates the position of the tip. The histogram in the right lower corner shows the average values of elasticity modulus Col6a1-/-, mdx, and wild type fibers. * significant difference compared to wild type, $\$$ significant difference compared to Col6a1-/-.

fibers showed a time constant similar to wild type fibers, but the model analysis indicated that such time constant value was the result of reduced values of both capacitance and conductance (Figures 5(e) and 5(f). Importantly, the instantaneous, time-independent resistance measured in the first $\mathrm{ms}$ after current injection was significantly lower in mutant than in wild type fibers. Thus, according to the model analysis, the higher membrane conductance of Col6a1-/fibers was due to a higher leakage and was associated with a lower membrane capacitance.

\subsection{Resting Potential and Action Potential Measured in Intact} FDB Muscle Preparations. In intact muscles the lack of Collagen VI creates a clear difference between mutant and wild type fibers which grow in the absence or, respectively, in the presence of Collagen VI. Such difference is lost in the culture dishes, where both mutant and wild type fibers are maintained in culture for 6 days after dissociation in the same environmental conditions (culture medium, adhesion substrate). In mdx muscles, a substantial subpopulation of fibers is severely damaged and necrotic and such fibers likely disappear during the dissociation procedure or in the first day in culture. To account for this and characterize the membrane properties of the fibers still embedded in the extracellular matrix, membrane conductance and excitability were measured also in fibers from FDB muscles dissected but kept intact "in vitro", without enzymatic dissociation. In full agreement with the measurements performed on cultured fibers, the membrane resting resistance was significantly reduced in Col6a1-/- and was also reduced in mdx muscle fibers compared to wild type muscle fibers (see Figure 6(b)), while the membrane resting potential was unaffected in Col6a $1-/-$ and significantly reduced in mdx (Figure 6(a)). Moreover, the action potential amplitude (Figure 6(c)) was not significantly different in Col6a1-/- with respect to wild type, while it was slightly but significantly increased in $\mathrm{mdx}$. The rate of depolarization showed a reduction in both mutant strains compared to wild type fibers (Figure 6(d)). 


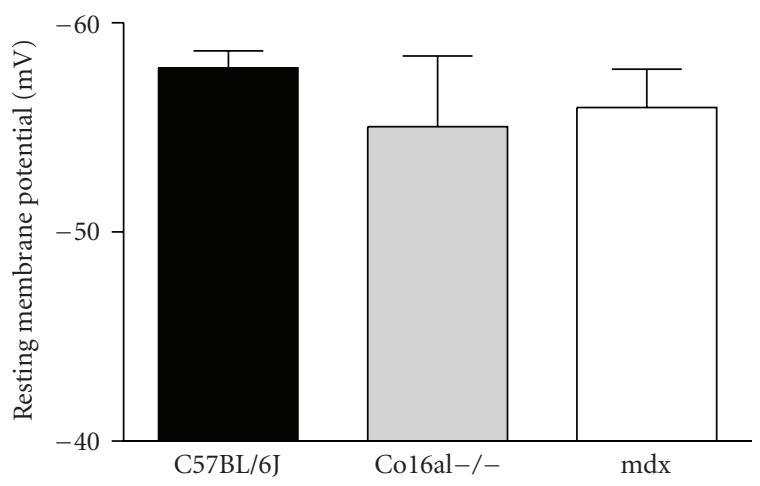

(a)

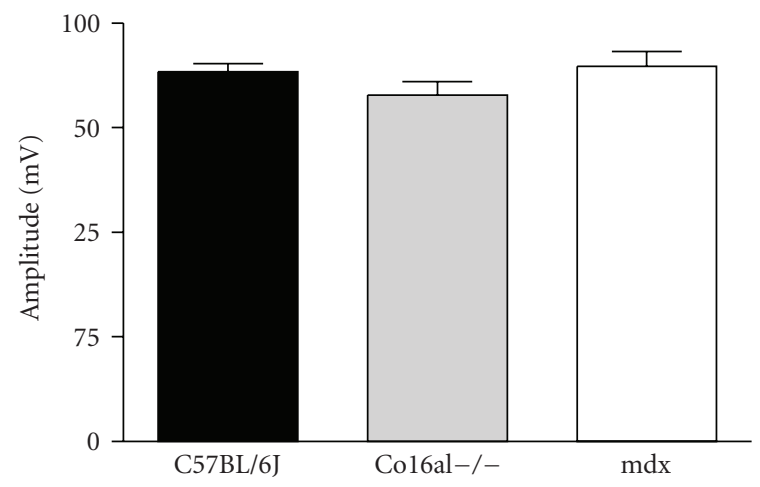

(c)

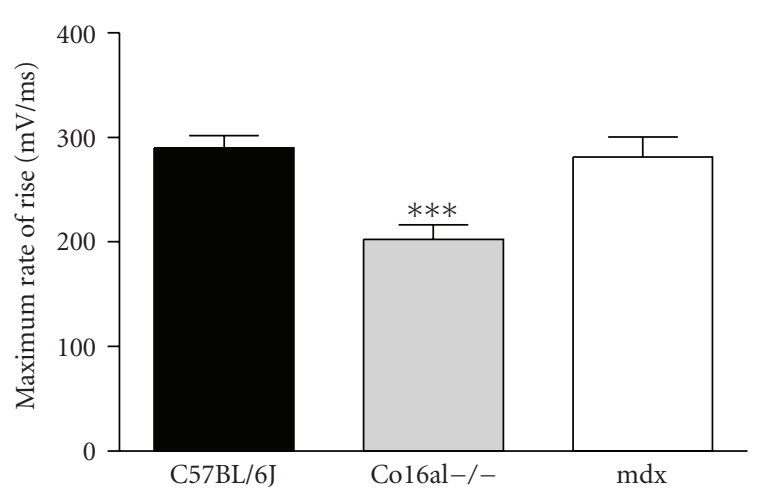

(b)

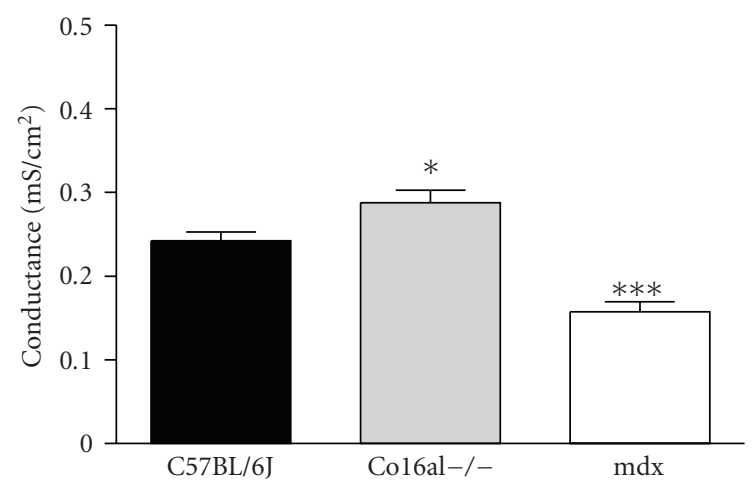

(d)

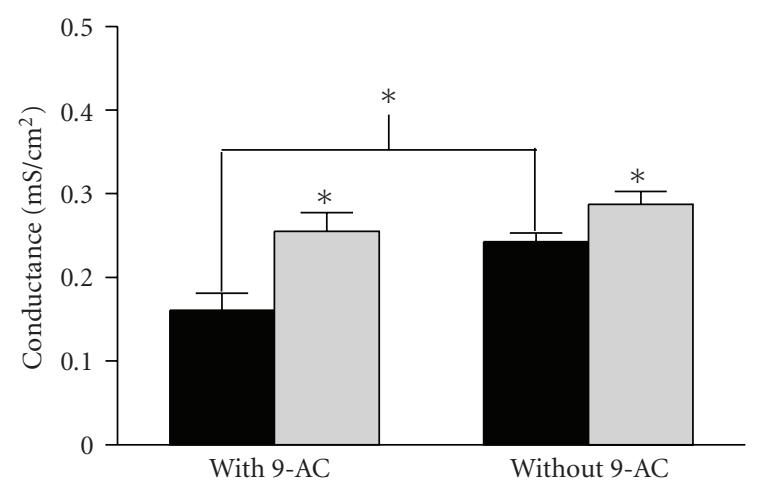

(e)

Figure 4: Passive and active membrane electrical properties of single muscle fibers kept in culture. Resting membrane potential (a), maximum rate of depolarization (b), action potential amplitude (c), and resting conductance determined with a hyperpolarizing step and normalized to sarcolemma surface of the wild type (black column), Col6a1-/- (grey column), mdx (white column). Mean and standard errors of measurements obtained in 66 wild type, 52 Col6a1-/ - and $32 \mathrm{mdx}$ fibers. ${ }^{*} P<.05$ and ${ }^{* * *} P<.001$ compared to wild type. (e) shows the determination of membrane conductance in a separate set of wild type (black column) and Col6a1-/- fibers (grey column) in the absence and in the presence of 9 -anthracene carboxylic acid $(9-\mathrm{AC}) .{ }^{*}$ denotes $P<.05$ both in comparison between the two fiber types and in comparison between absence and presence of 9-AC.

\section{Discussion}

The results obtained in the present study provide the first complete description of the biophysical properties of the sarcolemma of Col6a1-/- muscle fibers in comparison with wild type and mdx muscle fibers. The mutant fibers not only displayed alterations in membrane conductance and capacitance as well as in sarcolemma elasticity when compared to wild type muscle fibers but were also different from $\mathrm{mdx}$ muscle fibers. Importantly, such alterations were demonstrated in fibers in good conditions, able to survive for a long time in culture, and for this reason should not be considered expression of final degenerative processes, but more related to causal mechanisms of the muscle pathology. All experiments were carried out on FDB muscles which allow an easy dissociation of single fibers. FDB muscles are mainly composed of fast fibers [18] and, according to available data $[4,13]$, are clearly affected by 


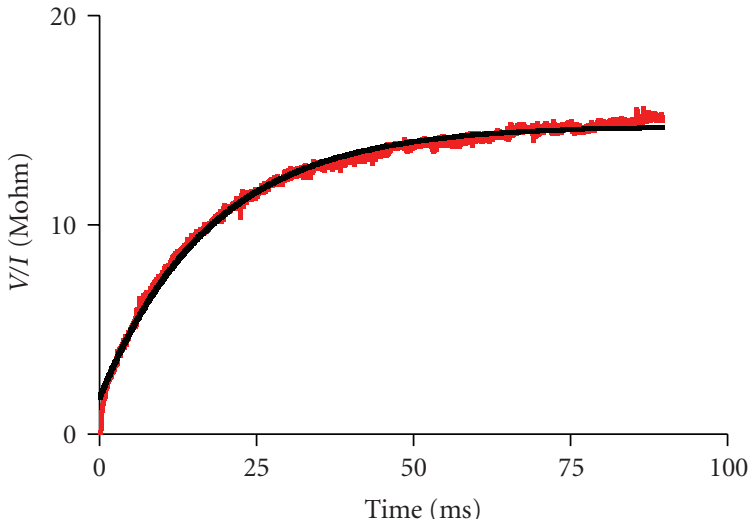

Data Co16a-/-

$\square$ Fit

(a)

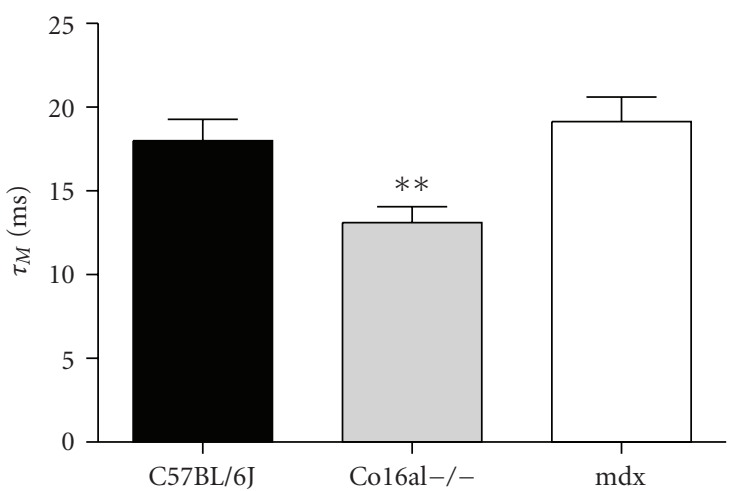

(c)

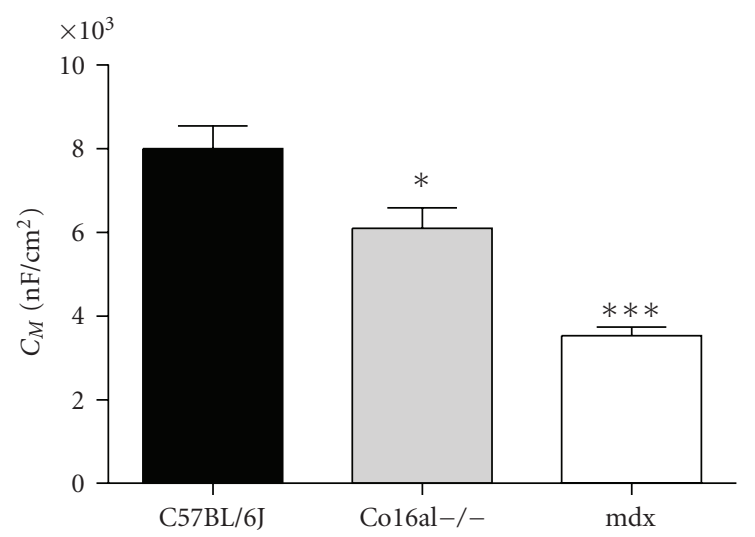

(e)

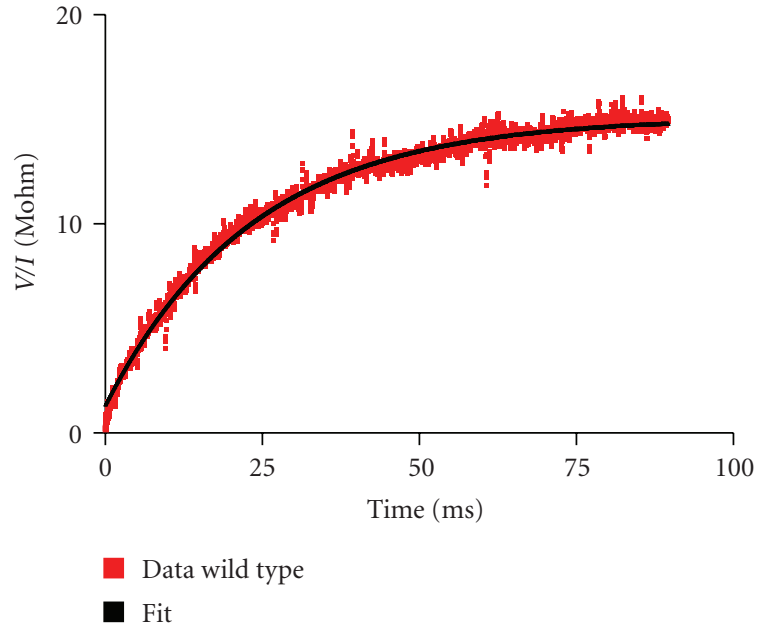

(b)

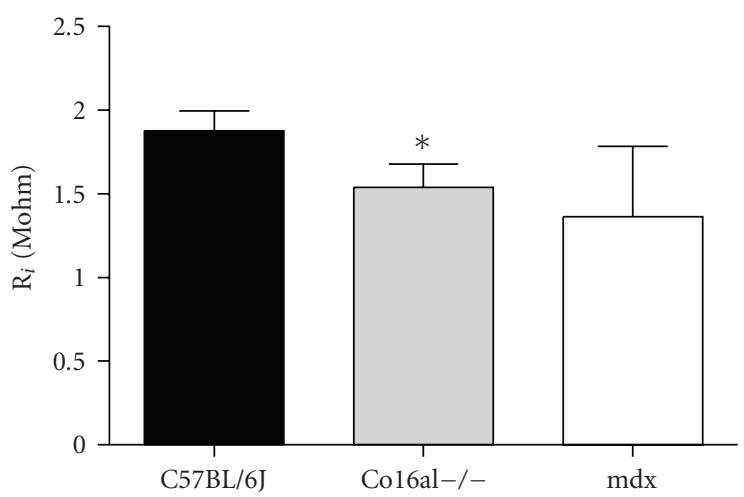

(d)

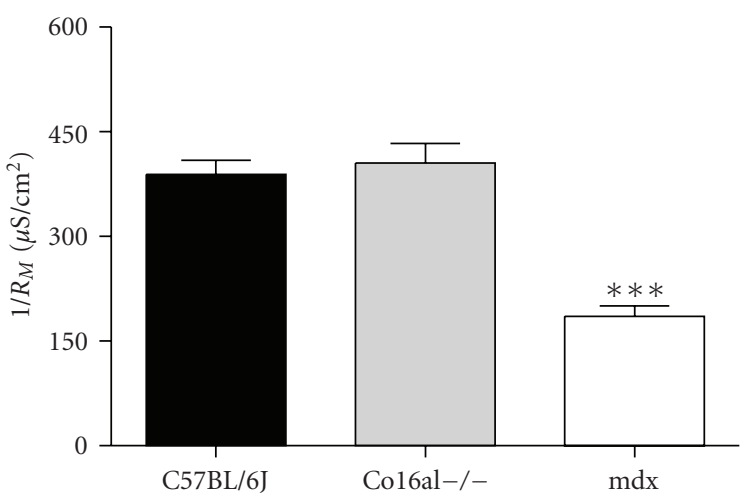

(f)

Figure 5: Analysis of resistance and capacity of the sarcolemma based on the kinetics of the membrane potential change during a hyperpolarizing step. Panels (a) and (b) show examples of fitting to the curves of membrane charging (expressed as the ratio V/I, membrane potential divided by injected current) with a step of hyperpolarizing current of $1 \mathrm{nA}$ from a holding potential of $-100 \mathrm{mV}$. Only the first $90 \mathrm{~ms}$, corresponding to 5000 data points are shown. Note the initial instantaneous jump in potential. The values of the parameters calculated with the analysis are shown in the histograms (c) - (f) as means and standard errors. Rm and Cm are the resistance and the capacity calculated by the model, their product being equal to the time constant $\tau$. Ri denotes the instantaneous, time-independent resistance. Wild type (black column), Col6a1-/- (gray columns), mdx (white columns) are reported in each histogram with ${ }^{*} P<.05, * * P<.01,{ }^{* * *} P<.001$ compared to wild type. The numbers of fibers studied is the same as in Figure 4. 


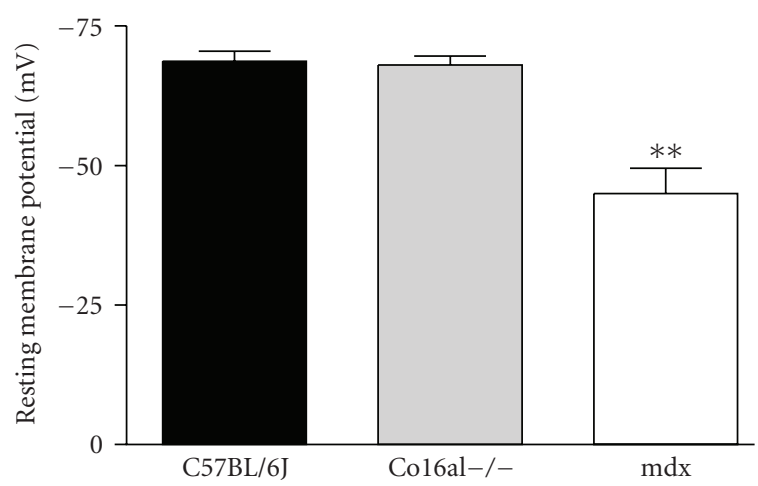

(a)

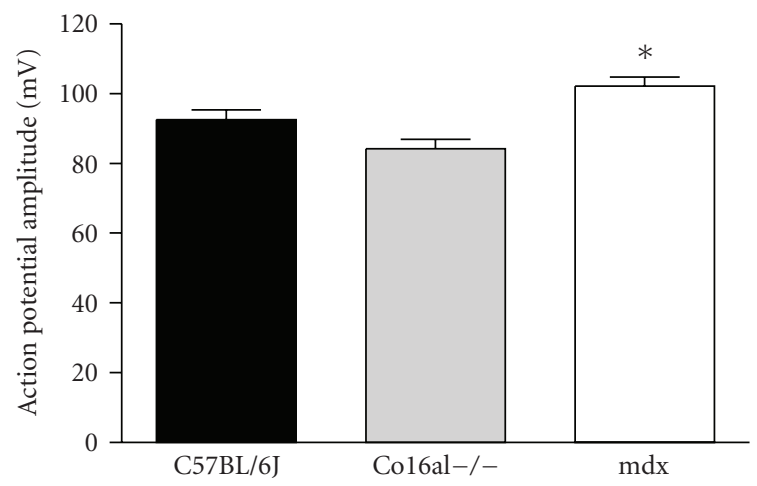

(c)

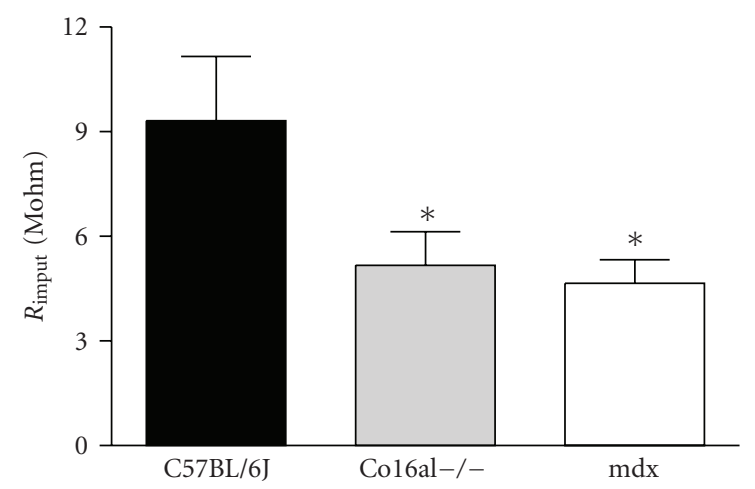

(b)

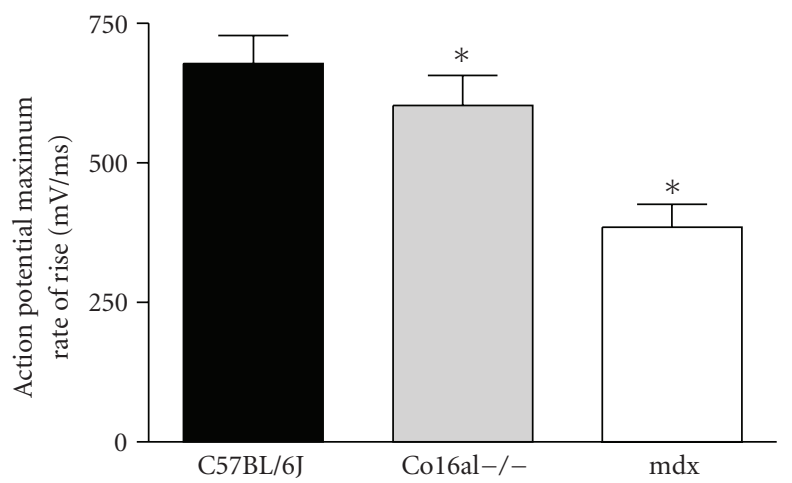

(d)

Figure 6: Passive and active membrane electrical properties of muscle fibers analysed in intact freshly dissected FDB muscle. Electrophysiological parameters determined in FDB fibers are shown as means with standard errors for wild type (black columns), Col6a1-/- (gray columns) and mdx (white columns) muscles. Histogram (a) shows resting membrane potential (rpm), histogram (b) shows input resistance, histogram (c) shows action potential amplitude, and histogram (d) shows rate of depolarization during action potential. ${ }^{*} P<.05$ and ${ }^{* *} P<.01$ compared to wild type.

the dystrophic process both in $\mathrm{mdx}$ and in Col6a1-/mice.

Our previous studies have shown that the lack of Collagen VI (COL VI) causes a mild dystrophy in mice [3, 4], characterized by an increased rate of apoptosis which leads slowly but progressively to fiber death. Random sampling of fibers from muscles of mice lacking COL VI yield three main populations of fibers: a majority of fibers apparently healthy, many fibers with some structural alterations but still able to contract, and a few fibers positive to Evans Blue Dye (EBD), completely altered in their structure and unable of contractile response [4]. Enzymatic dissociation of FDB produced only fibers apparently healthy, likely because all damaged fibers were destroyed during dissociation. Such apparently healthy fibers, however, when put in culture and challenged with olygomycin, revealed a latent mitochondrial weakness likely causally related to apoptosis triggering mechanism [4]. Cyclosporin A, an inhibitor of the opening of the mitochondrial permeability transition pore, was effective in reducing apoptosis rate in murine and human myopathies due to lack of COLVI and this gives support to the causal role of the mitochondria $[4,5]$. Muscle dystrophy in $\mathrm{mdx}$ mice shows a very different picture characterized by a high proportion of necrotic EBD positive fibers $[19,20]$ and a high proportion of newly formed regenerating fibers. Such fibers likely disappear during the dissociation procedure and the first day in culture, but the surviving fibers still bear clear alterations of the sarcolemma which could be detected in this study. The choice of performing experiments on the sixth day after dissociation was aimed to restrict the comparison to fibers in good conditions. Thus, the alterations observed in the sarcolemma could not be attributed to the final degenerative process, but could be safely considered expression of the initial pathogenetic process. The diversity between the severe selection occurring in the culture of $\mathrm{mdx}$ muscle fibers and the greater stability of Col6a $1-/-$ muscle fiber culture suggested to repeat the electrophysiological measurements also in intact freshly dissected FDB muscles, where a complete, unselected fiber population could be sampled.

Compared to wild type, Col6a1-/- muscle fibers displayed an increased resting conductance, detectable in fibers dissociated from FDB and kept in culture for 6 days as well as in fibers imbedded in freshly dissected FDB muscles. The first explanation considered was a change in chloride conductance, since chloride conductance is predominant in resting muscle membrane [16] and variations have been reported in relation to fiber type and pathological conditions [21]. 
Experiments with 9-Anthracene, a known blocker of chloride channels [17], lead to discard this hypothesis. Moreover, the analysis of the time course of the membrane charging after the step injection of a hyperpolarizing current showed that the difference in membrane conductance was mainly attributable to the initial time-independent component of membrane conductance. Such component was identified with a membrane leakage, possibly related to membrane fragility. Another finding plays in favour of an aspecific ionic leakage which might allow entrance of positive ions. Actually, the rate of rise of action potential was significantly decreased in Col6a1-/- muscle fibers. The rate of depolarization during action potential is determined by the opening of sodium channels and the related inward current. Thus, a likely explanation is that a conserved channel density is accompanied by a reduced sodium concentration gradient which fits very well with the increased membrane leakage. Resting membrane potential however was not changed and this would require an increased potassium current at rest which might also fit with the reduced weight of chloride currents in mutant fibers.

Mdx fibers in culture showed a decreased membrane conductance compared to wild type and Col6a1-/- muscle fibers. The finding was confirmed by the model analysis and is in agreement with previous observations [11] which linked such decrease to a reduced chloride conductance. Interestingly, the electrophysiological analysis of the intact freshly dissected FDB muscle gave an opposite indication, that is, a decreased membrane resistance. The likely explanation is given by the presence of a high number of pathological and deeply altered fibers in the population impaled with the microelectrodes in the intact muscle. The diversity between the features of the mdx fibers in culture compared to those in the intact muscle is also confirmed by the reduction of the resting membrane potential and of the rate of depolarization during action potential, two features present in intact muscle and not present in cultured fibers.

The aspecific leakage observed in Col6a1-/- muscle fibers is suggestive of structural alterations of the sarcolemma. ECM proteins may play the role of organizer of the sarcolemma structure, as recently shown for biglycan [22] and their absence might induce structural alterations. The sarcolemma topography analysed with AFM (Atomic Force Microscopy), with a resolution comparable to the transmission electron microscopy (TEM) [15], did not provide, however, any clue in favour of such structural alterations in general agreement with previous studies with TEM, showing mitochondria and SR swelling but not sarcolemma disorganization [4]. AFM experiments, however, revealed a reduced membrane stiffness (Young modulus reduced by 30\%) and the electrical capacitance, which is related to the membrane surface, was also found reduced (by 20\%). Both Young modulus and capacitance were "normalized" to sarcolemma surface, calculated from optical microscopy imaging with the resolution of $\mu \mathrm{m}$. However, the "true" membrane surface should also account for $\mathrm{T}$ tubules, caveolae, and foldings. It is thus possible that the "true" sarcolemma surface is changed in a way which is not quantified even with the high resolution achieved by AFM. As far as Young modulus reduction must be taken into account that the indentation reached $100 \mathrm{~nm}$ and, therefore, some subsarcolemmal mitochondria might contribute to the calculated elasticity. Mitochondrial swelling was reported in our previous study [4].

The increased Young modulus in $\mathrm{mdx}$ fibers in culture was an unexpected result. Early attempts to determine the stiffness of the sarcolemma in mdx muscle dystrophy were carried out mainly in myotubes with various techniques (aspiration in micropipette [23], osmotic swelling [24], indentation with glass probes [25]) and with uncertain results. Few months ago, the results of measurements of sarcolemma stiffness on adult mdx fibers with AFM were published [12] and showed that stiffness was about $1 / 3$ in $\mathrm{mdx}$ compared to wild type fibers. The disagreement between the results obtained in this study and those reported by [12] can find more than one explanation: (i) FDB fibers surviving in culture were examined in this study, while an unselected population of Tibialis anterior fibers was tested by Puttini and coworkers [12], (ii) the indentation amplitude was $500 \mathrm{~nm}$ in the experiments of Puttini and coworkers [12], while in this study the amplitude was only $100 \mathrm{~nm}$ and only the unloading curve was considered. It is important to recall that there are recent indications of changes in subsarcolemmal viscosity of $\mathrm{mdx}$ muscle fibers likely due to actin cytoskeleton reorganization [26] and that the distance between sarcolemma and superficial mitochondria or myofibrils is generally in the order of tenths of $\mu \mathrm{m}$, and (iii) the sarcomere length of the fibers in culture was low (see below), while it was controlled in the experiments of Puttini and coworkers [12], it is likely that the sarcolemmal stiffness increases with sarcomere length in relation with the reduction of the "membrane reservoir" given by minor foldings and caveolae as suggested by Dulhunty and Franzini-Armstrong [27]. The topography of the sarcolemma as detected by AFM was not substantially altered in mdx as well as Col6a1-/- muscle fibers when compared to wild type. The same folding periodicity could be observed and, if the greater foldings correspond to $\mathrm{Z}$ lines and to costameres as demonstrated by our previous study [15], the costamere structure is clearly preserved also in $\mathrm{mdx}$ muscle fibers, in spite of the lack of dystrophin. The lower folding likely corresponding to the $\mathrm{M}$ line was also detectable in mutant muscle fibers.

Differences in sarcomere length were also observed between mutant and wild type fibers and might represent an indication of subtle structural differences. Dissociated fibers attach to the laminin substrate without any constraint at their slack length, which is set in the first place by myofibrillar proteins such as titin [28-30]. There are, however, no reasons to believe that the shorter sarcomere length observed in Col6a1-/- and mdx fibers is an indication of a difference in myofibrillar proteins. A contribution of the sarcolemma cannot be excluded either in terms of the mechanical alterations (see above Young Modulus) or in connection to a fiber swelling related to the changes in sarcolemma permeability.

In conclusion, the results obtained show that muscle fibers prepared from mice bearing two distinct types of muscle dystrophy, kept in culture and apparently in good 
functional and structural conditions, exhibit clear sarcolemmal alterations which are likely related with the molecular defects responsible for the dystrophy. For mdx fibers, the present data allow the description of a set of fibers lacking dystrophin but apparently in good health, not affected by a progressive necrosis process and not regenerating. Such fibers show some previously demonstrated features, such as the reduced membrane conductance, but also reveal other interesting aspects such as the increased stiffness and the reduced membrane capacity. For Col6a1-/- the reduced membrane stiffness and the increased leakage are suggestive of an initial sarcolemmal disorganization which might represent the still missing link between the lack of an important extracellular protein, such COL VI, and the well investigated mitochondrial dysfunction. The control of the opening of the mitochondrial permeability transition pore (PTP) has been the target of the attempts to treat the human muscle disease caused by mutations of COL VI, Bethlem myopathy, and Ulrich congenital dystrophy $[4,5]$. The understanding of the pathway connecting COL VI in the extracellular matrix and mitochondria can open a new perspective to develop more effective treatments of the disease. The indications, emerging from the present study, of sarcolemma alterations may represent a step in this direction.

\section{Acknowledgment}

The study was supported by Telethon Grant GGP04113.

\section{References}

[1] G. J. Jöbsis, J. M. Boers, P. G. Barth, and M. de Visser, "Bethlem myopathy: a slowly progressive congenital muscular dystrophy with contractures," Brain, vol. 122, no. 4, pp. 649-655, 1999.

[2] O. Camacho Vanegas, E. Bertini, R.-Z. Zhang, et al., "Ullrich scleroatonic muscular dystrophy is caused by recessive mutations in collagen type VI," Proceedings of the National Academy of Sciences of the United States of America, vol. 98, no. 13, pp. 7516-7521, 2001.

[3] P. Bonaldo, P. Braghetta, M. Zanetti, S. Piccolo, D. Volpin, and G. M. Bressan, "Collagen VI deficiency induces early onset myopathy in the mouse: an animal model for Bethlem myopathy," Human Molecular Genetics, vol. 7, no. 13, pp. 2135-2140, 1998.

[4] W. A. Irwin, N. Bergamin, P. Sabatelli, et al., "Mitochondrial dysfunction and apoptosis in myopathic mice with collagen VI deficiency," Nature Genetics, vol. 35, no. 4, pp. 367-371, 2003.

[5] A. Angelin, T. Tiepolo, P. Sabatelli, et al., "Mitochondrial dysfunction in the pathogenesis of Ullrich congenital muscular dystrophy and prospective therapy with cyclosporins," Proceedings of the National Academy of Sciences of the United States of America, vol. 104, no. 3, pp. 991-996, 2007.

[6] E. Werner and Z. Werb, "Integrins engage mitochondrial function for signal transduction by a mechanism dependent on Rho GTPases," Journal of Cell Biology, vol. 158, no. 2, pp. 357-368, 2002.

[7] S. Petrini, A. Tessa, W. B. Stallcup, et al., "Altered expression of the MCSP/NG2 chondroitin sulfate proteoglycan in collagen VI deficiency," Molecular and Cellular Neuroscience, vol. 30, no. 3, pp. 408-417, 2005.
[8] U. Mayer, "Integrins: redundant or important players in skeletal muscle?" Journal of Biological Chemistry, vol. 278, no. 17, pp. 14587-14590, 2003.

[9] R. Barresi and K. P. Campbell, "Dystroglycan: from biosynthesis to pathogenesis of human disease," Journal of Cell Science, vol. 119, no. 2, pp. 199-207, 2006.

[10] S. H. Gee, R. Madhavan, S. R. Levinson, J. H. Caldwell, R. Sealock, and S. C. Froehner, "Interaction of muscle and brain sodium channels with multiple members of the syntrophin family of dystrophin-associated proteins," Journal of Neuroscience, vol. 18, no. 1, pp. 128-137, 1998.

[11] A. De Luca, S. Pierno, and D. Conte Camerino, "Electrical properties of diaphragm and EDL muscles during the life of dystrophic mice," American Journal of Physiology, vol. 272, no. 1, pp. C333-C340, 1997.

[12] S. Puttini, M. Lekka, O. M. Dorchies, et al., "Gene-mediated restoration of normal myofiber elasticity in dystrophic muscles," Molecular Therapy, vol. 17, no. 1, pp. 19-25, 2009.

[13] S. I. Head, "Membrane potential, resting calcium and calcium transients in isolated muscle fibres from normal and dystrophic mice," Journal of Physiology, vol. 469, pp. 11-19, 1993.

[14] S. Pierno, M. P. Didonna, V. Cippone, et al., "Effects of chronic treatment with statins and fenofibrate on rat skeletal muscle: a biochemical, histological and electrophysiological study," British Journal of Pharmacology, vol. 149, no. 7, pp. 909-919, 2006.

[15] E. Defranchi, E. Bonaccurso, M. Tedesco, et al., "Imaging and elasticity measurements of the sarcolemma of fully differentiated skeletal muscle fibres," Microscopy Research and Technique, vol. 67, no. 1, pp. 27-35, 2005.

[16] T. J. Jentsch, V. Stein, F. Weinreich, and A. A. Zdebik, "Molecular structure and physiological function of chloride channels," Physiological Reviews, vol. 82, no. 2, pp. 503-568, 2002.

[17] P. T. Palade and R. L. Barchi, "Characterization of the chloride conductance in muscle fibers of the rat diaphragm," The Journal of General Physiology, vol. 69, pp. 325-342, 1977.

[18] E. González, M. L. Messi, Z. Zheng, and O. Delbono, "Insulinlike growth factor-1 prevents age-related decrease in specific force and intracellular $\mathrm{Ca}^{2+}$ in single intact muscle fibres from transgenic mice," Journal of Physiology, vol. 552, no. 3, pp. 833844, 2003.

[19] P. Moens, P. H. W. W. Baatsen, and G. Maréchal, "Increased susceptibility of EDL muscles from mdx mice to damage induced by contractions with stretch," Journal of Muscle Research and Cell Motility, vol. 14, no. 4, pp. 446-451, 1993.

[20] B. J. Petrof, J. B. Shrager, H. H. Stedman, A. M. Kelly, and H. L. Sweeney, "Dystrophin protects the sarcolemma from stresses developed during muscle contraction," Proceedings of the National Academy of Sciences of the United States of America, vol. 90, no. 8, pp. 3710-3714, 1993.

[21] S. Pierno, J.-F. Desaphy, A. Liantonio, et al., "Change of chloride ion channel conductance is an early event of slowto-fast fibre type transition during unloading-induced muscle disuse," Brain, vol. 125, no. 7, pp. 1510-1521, 2002.

[22] M. L. Mercado, A. R. Amenta, H. Hagiwara, et al., "Biglycan regulates the expression and sarcolemmal localization of dystrobrevin, syntrophin, and nNOS," The FASEB Journal, vol. 20, no. 10, pp. 1724-1726, 2006.

[23] O. F. Hutter, F. L. Burton, and D. L. Bovell, "Mechanical properties of normal and mdx mouse sarcolemma: bearing on function of dystrophin," Journal of Muscle Research and Cell Motility, vol. 12, no. 6, pp. 585-589, 1991. 
[24] A. Menke and H. Jockusch, "Decreased osmotic stability of dystrophin-less muscle cells from the mdx mouse," Nature, vol. 349, no. 6304, pp. 69-71, 1991.

[25] C. Pasternak, S. Wong, and E. L. Elson, "Mechanical function of dystrophin in muscle cells," Journal of Cell Biology, vol. 128, no. 3, pp. 355-361, 1995.

[26] T. M. Suchyna and F. Sachs, "Mechanosensitive channel properties and membrane mechanics in mouse dystrophic myotubes," Journal of Physiology, vol. 581, no. 1, pp. 369-387, 2007.

[27] A. F. Dulhunty and C. Franzini-Armstrong, "The passive electrical properties of frog skeletal muscle fibres at different sarcomere lengths," Journal of Physiology, vol. 266, no. 3, pp. 687-711, 1977.

[28] K. Wang, R. McCarter, J. Wright, J. Beverly, and R. RamirezMitchell, "Regulation of skeletal muscle stiffness and elasticity by titin isoforms: a test of the segmental extension model of resting tension," Proceedings of the National Academy of Sciences of the United States of America, vol. 88, no. 16, pp. 7101-7105, 1991.

[29] S. Labeit and B. Kolmerer, "Titins: giant proteins in charge of muscle ultrastructure and elasticity," Science, vol. 270, no. 5234, pp. 293-296, 1995.

[30] N. Preetha, W. Yiming, M. Helmes, F. Norio, L. Siegfried, and H. Granzier, "Restoring force development by titin/connectin and assessment of Ig domain unfolding," Journal of Muscle Research and Cell Motility, vol. 26, no. 6-8, pp. 307-317, 2005. 

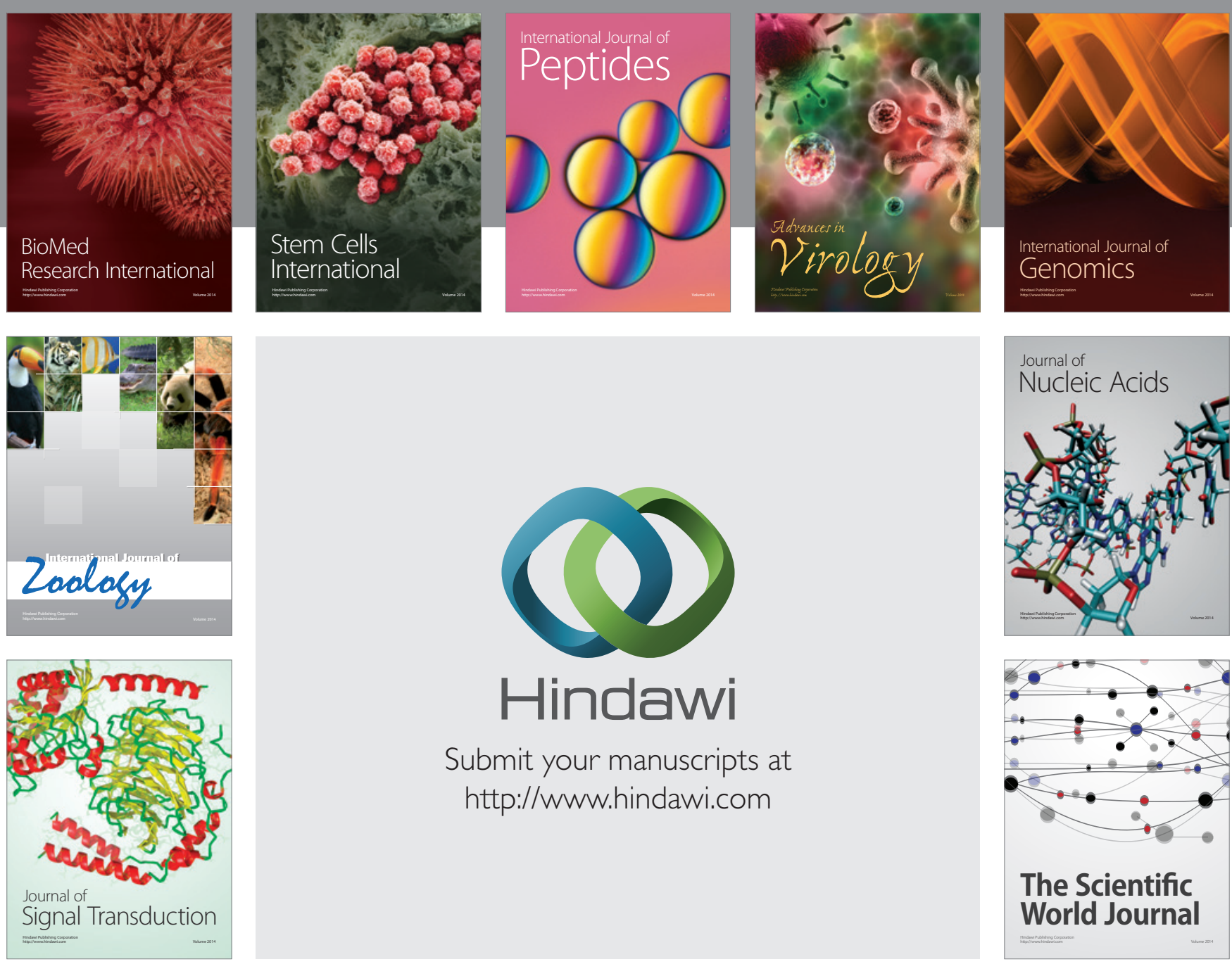

Submit your manuscripts at

http://www.hindawi.com
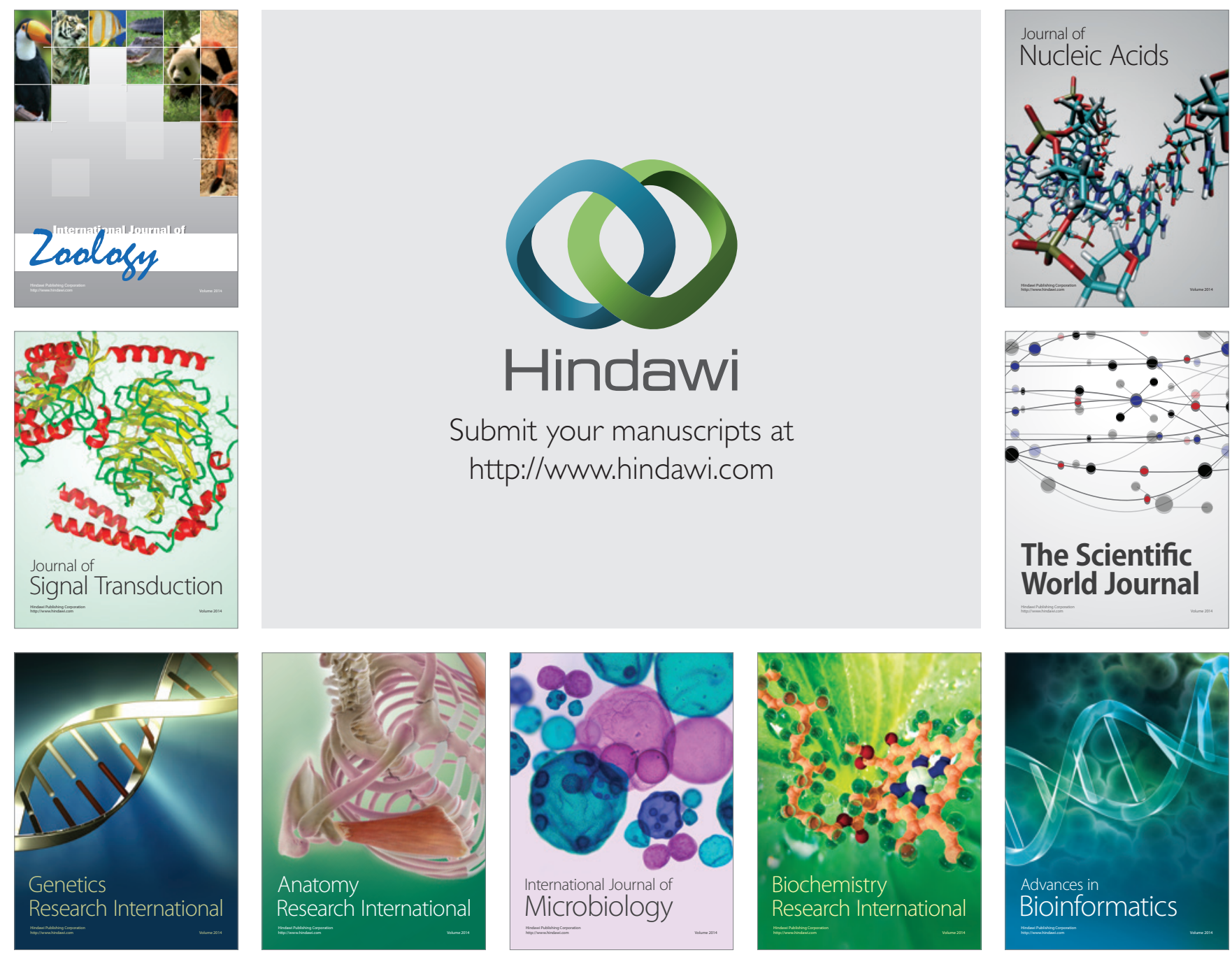

The Scientific World Journal
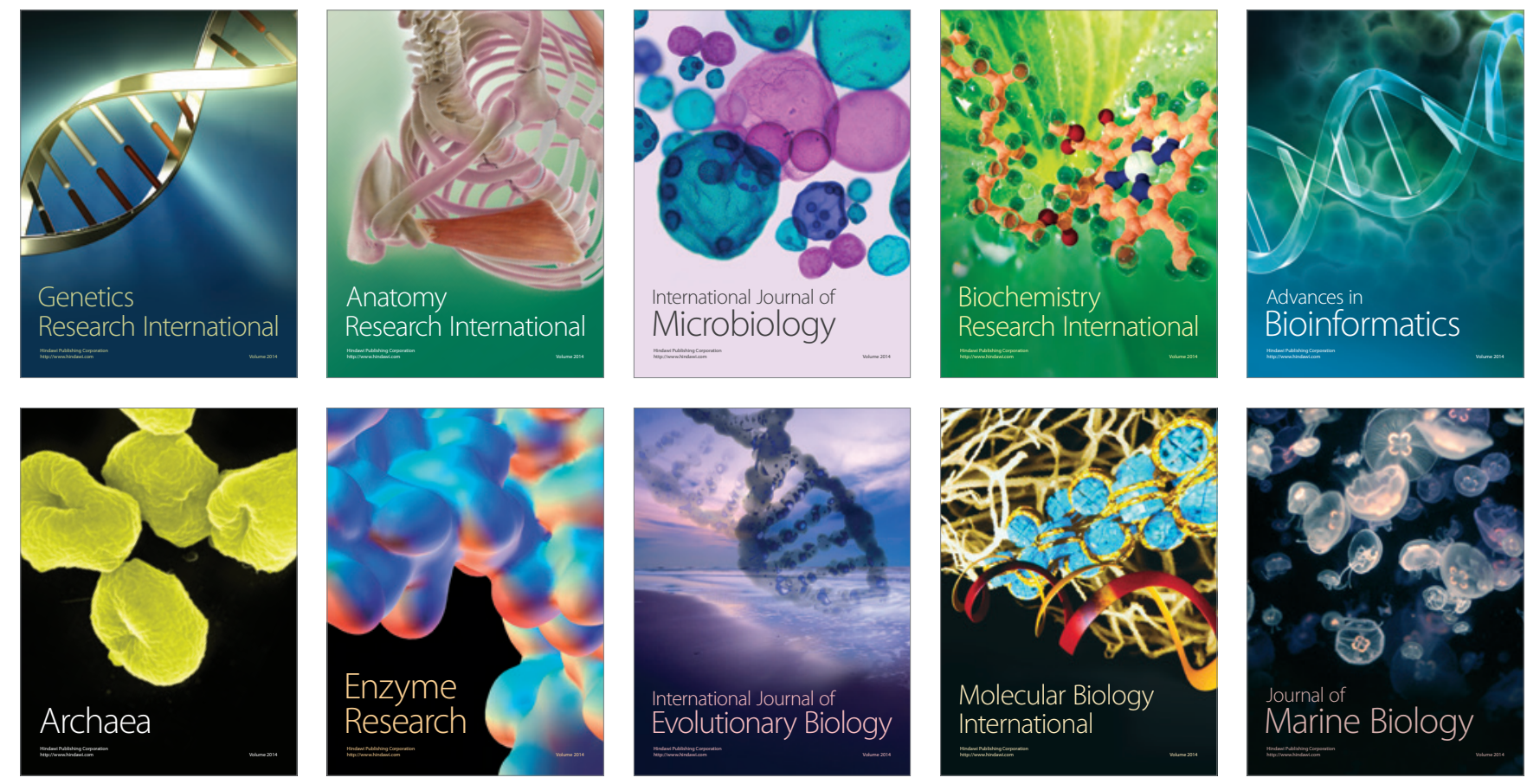